\title{
Long-term incidence of depression and predictors of depressive symptoms in older stroke survivors
}

Louise M. Allan, Elise N. Rowan, Alan J. Thomas, Tuomo M. Polvikoski, John T. O'Brien and Raj N. Kalaria

\section{Background}

Depression is common and an important consequence of stroke but there is limited information on the longer-term relationship between these conditions.

\author{
Aims \\ To identify the prevalence, incidence and predictors of \\ depression in a secondary-care-based cohort of stroke \\ survivors aged over 75 years, from 3 months to up to 10 \\ years post-stroke.
}

\section{Method}

Depression was assessed annually by three methods: major depression by DSM-IV criteria, the self-rated Geriatric Depression Scale (GDS) and the observer-rated Cornell scale.

\section{Results}

We found the highest rates, $31.7 \%$ baseline prevalence, of depressive symptoms with the GDS compared with 9.7\% using the Cornell scale and 1.2\% using DSM-IV criteria. Incidence rates were 36.9, 5.90 and 4.18 episodes per 100 person years respectively. Baseline GDS score was the most consistent predictor of depressive symptoms at all time points in both univariate and multivariate analyses. Other predictors included cognitive impairment, impaired activities of daily living and in the early period, vascular risk factor burden and dementia.

\section{Conclusions}

Our results emphasise the importance of psychiatric follow-up for those with early-onset post-stroke depression and long-term monitoring of mood in people who have had a stroke and remain at high risk of depression.

\section{Declaration of interest}

None.
Psychiatrists, especially those working in liaison or old age psychiatry, frequently encounter people who have had a stroke and mood change, especially depression, is a well-recognised sequel. Estimates of frequency varying from 25 to $36 \%$ in two previous meta-analyses, ${ }^{1,2}$ depending on the setting, population studied, definition of depression and timing of assessments. A weakness in the current literature is the relatively short-term nature of follow-up and we therefore have a limited understanding of the longer-term risk of depression after stroke and its predictors, yet most psychiatric assessments after stroke occur years rather than months later. Ayerbe et al found only eight studies that had longer-term follow-up beyond 1 year poststroke, ${ }^{3}$ which included two population-based cohorts that had followed participants up to 5 years ${ }^{3,4}$ but no hospital-based cohorts that had such a long period of follow-up, and there are no reports of follow-up beyond 5 years. There is also a paucity of well-designed studies large enough to examine predictors of depression after stroke, and only four of these studies have followed up participants for longer than 1 year. ${ }^{5}$ Major predictors of post-stroke depression include disability, pre-stroke depression and cognitive impairment, and thus people with these factors may benefit from proactive monitoring of mood in the first year of post-stroke care. ${ }^{6}$ Since acute stroke units have resulted in improved survival after stroke, many older people survive far beyond this phase, so there is an additional issue about whether longer-term follow-up, and monitoring of both mood and cognition, would be beneficial in preventing progression of cognitive decline, deterioration in mood and functional ability. In designing randomised controlled trials to identify appropriate strategies for the long-term prevention of such deterioration, there is also a need to understand its underlying aetiology. The vascular hypothesis postulates that some depression in older people is a vascular brain disease. If this is true, then stroke patients will be at increased risk of depression after stroke, but those at greatest risk will be those with a greater burden of vascular disease, reflected in an increased number of vascular risk factors including ischaemic heart disease, peripheral vascular disease, diabetes mellitus, smoking and atrial fibrillation. ${ }^{7}$ We have previously shown that a greater burden of vascular factors is associated with a greater likelihood of clinical expression of a dementia syndrome in the long term after stroke in older people. ${ }^{8}$ Whereas depression predicted the incidence of dementia in univariate analyses, it was the number of cardiovascular risk factors that was retained in multivariate analyses, along with cognitive impairment. It is therefore likely that mood disorders and cognitive decline after stroke share a common relationship with both burden of vascular risk factors and underlying neuropathology. The demonstration of any association between mood changes and vascular risk factors or vascular pathology would be important, since depression is a recognised risk factor for dementia (both Alzheimer's disease and vascular dementia), but the nature of the link remains unclear.

In this study, we examine depression in the COGFAST cohort, which is a secondary-care-based longitudinal study of older people (aged over 75) who have had a stroke, with follow-up data of up to 10 years and a brain tissue donation programme. ${ }^{8}$ The cohort provides a rare opportunity to look at predictors of mood disorders in the very long term after stroke, and also to examine their associations with neuropathology. We hypothesised that, in accordance with the vascular hypothesis of depression, an increased burden of vascular risk factors would predict depressive symptoms in the long term. We further hypothesised that those with the greatest degree of cognitive decline would account for the majority of incident depression, and that such individuals would have a greater burden of vascular neuropathology at post-mortem. 


Method
Study design and participants

Older stroke patients $(n=706) \geqslant 75$ years were screened consecutively from hospital-based stroke registers in Tyneside and Wearside in the North East of England (Fig. 1). Potential participants were evaluated at least 3 months after stroke (on the basis of the design of Desmond et $a l^{9}$ and to enable resolution of acute poststroke delirium), with a standardised battery comprised of medical history, Mini-Mental State Examination (MMSE) ${ }^{10}$ score, assessment of neurological deficits, a blood screen and a review of the computed tomography (CT) brain scan undertaken at the time of the stroke. Stroke was defined according to the World Health Organization definition. ${ }^{11}$ We excluded those individuals with stroke (a) who were younger than 75 years, (b) with significant physical illness and disabilities that precluded neuropsychological evaluation (such as visual impairment, aphasia, hemiparesis affecting the hand used for writing), (c) who had an MMSE score less than 24 at the 3-month assessment, (d) had a diagnosis of dementia according to DSM-III-R criteria ${ }^{12}$ or (e) declined to take part. Following general practitioner approval and discussion of the study, 355 of the original 706 patients were eligible for the post-stroke survivor study (Fig. 1). The characteristics of the full cohort have been described previously. ${ }^{13,14}$ In addition to this full cohort, a subgroup of 35 of the stroke survivors who already had dementia at baseline were recruited into a study of falls in dementia that was ongoing within the same department. ${ }^{15}$ These patients received the same follow-up protocol as the main cohort (described below).

Ethical approval was obtained from the Joint Ethics Committee of Newcastle and North Tyneside Health Authority, the University of Newcastle upon Tyne and the University of Northumbria at Newcastle, and participants gave written informed consent in accordance with the declaration of Helsinki.

\section{Baseline and follow-up assessments}

All participants completed a standardised baseline medical and neuropsychological assessment at 3 months post-stroke. Medical histories taken from the participants were supported by review of hospital charts. Data on putative predictors of depression included pre-stroke factors, stroke characteristics and factors related to outcome at 3 months. Pre-stroke factors were age, gender, current treatment for depression, diagnosis of previous stroke, hypertension (a documented history of blood pressure greater than $140 / 90 \mathrm{mmHg}$ or treatment of hypertension), atrial fibrillation, ischaemic heart disease, peripheral vascular disease, hypercholesterolaemia, diabetes (documented or treated), history of smoking prior to stroke and apolipoprotein genotype determined as described previously. ${ }^{16}$

Strokes were classified using the Oxford Community Stroke project (OCSP) classification. ${ }^{17}$ Stroke outcome factors were activities of daily living (assessed with the Bristol scale), ${ }^{18}$ cognition (assessed with the Cambridge Examination for Mental Disorders of the Elderly, section B (Cambridge Cognitive Examination, CAMCOG) ${ }^{19}$ which is a standardised paper and pencil test (maximum score, 107) for global cognitive performance), presence of mild cognitive impairment (based on Petersen criteria: ${ }^{20}$ those with cognitive scores $>1.5$ standard deviations below an age-matched control group were defined as having amnestic mild cognitive impairment (CAMCOG memory subscore $\leqslant 13$ ) and/or non-amnestic mild cognitive impairment (CAMCOG executive subscore $\leqslant 17)$ ), and finally severity of baseline depressive symptomatology. Severity of baseline depressive symptoms was quantified using the self-rated 15-item Geriatric Depression Scale (GDS). ${ }^{21}$

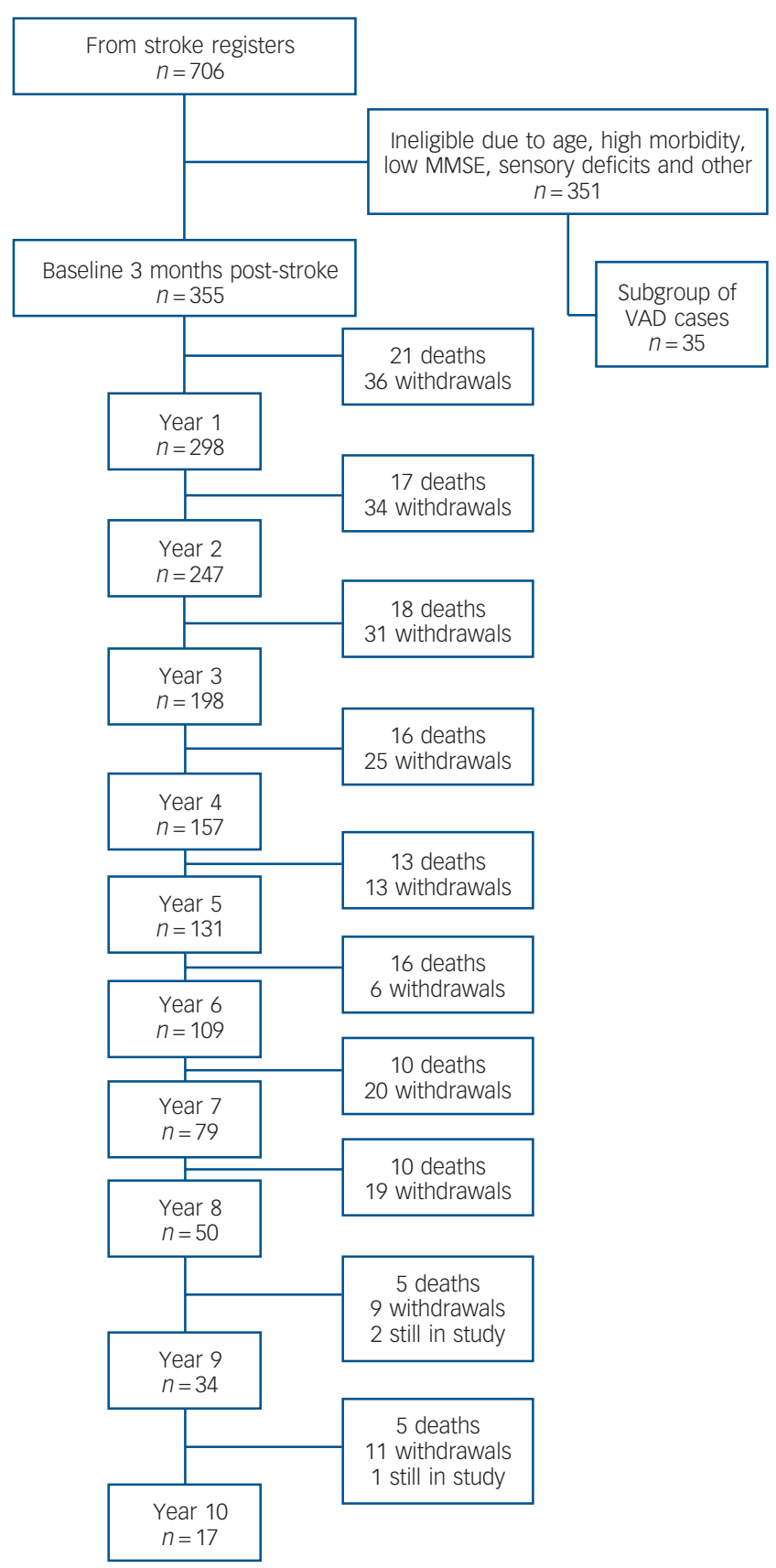

Fig. 1 Flow chart of participant follow-up.

Where totals show 'still in study', this refers to individuals who have so far completed less than 10 years of follow-up but who have not yet died or withdrawn from the less than 10 years of follow-up but who have not yet died or withdrawn
study. MMSE, Mini-Mental State Examination; VAD, vascular dementia.

Prevalent depression was defined by three methods: major depression according to the DSM-IV criteria ${ }^{22}$ assessed by a clinical research nurse or assistant psychologist, significant depressive symptoms defined as a score of $\geqslant 5$ on the GDS (GDS depression) or depressive disorder defined as a score of $\geqslant 10$ on the observerrated Cornell scale (Cornell depression). ${ }^{23}$ In keeping with other studies in older people, and to facilitate cross-study comparison, we utilised GDS score as our primary depression outcome for analysis. $^{24,25}$ As GDS scores are skewed, for analysis we converted scores to quartiles, based on GDS scores at baseline in the whole group.

Neuropsychological assessment was repeated annually, as in the baseline assessment, during a follow-up period of up to 10 years. 
Incident dementia according to DSM-IV criteria was assessed according to methods we have described previously. Incident depression was defined as described for prevalent depression.

\section{Neuropathological examination and dementia diagnoses}

Brains were retrieved from a total of 56 stroke survivors who came to autopsy. Six of these had dementia at baseline (Fig. 1) but were included in the assessment for comparative purposes. In the majority of cases bronchopneumonia was recorded as the cause of death.

Macroscopic and microscopic pathology was assessed with standardised protocols as described in Kalaria et al ${ }^{26}$ and Ihara et $a l^{27}$ Briefly: macroscopic infarcts were detected by visual inspection while dissecting the brain, and their presence was subsequently confirmed by microscopy. Haemotoxylin and eosin was used as the standard stain for general neuropathological assessment of the structure of the brain, and for confirmation/ detection of the infarcts. In this study, any infarct less than $5 \mathrm{~mm}$ in diameter was defined as a microinfarct. Gallyas and Bielschowsky's silver impregnation and tau immunohistochemistry were applied to assess neuritic plaques and neurofibrillary tangles for the 'CERAD' (Consortium to Establish a Registry for Alzheimer's Disease) plaque score ${ }^{28}$ and 'Braak and Braak' neurofibrillary tangle staging. ${ }^{29}$ Additional staining included $\alpha$-synuclein, ubiquitin and TDP-43 immunohistochemistry.

A clinical diagnosis of whether the dementia syndrome was present was made independently of neuropathological data prior to monthly clinicopathological consensus meetings where clinicians met with the pathologists to designate a final diagnosis for autopsied individuals. In all cases, additional pathologies such as that in Alzheimer's disease and dementia with Lewy bodies were noted. The pathological diagnosis of vascular dementia was then assigned if there was clinical evidence of dementia (DSM-IV) and the presence of multiple or cystic infarcts involving cortical and subcortical structures, border-zone infarcts, lacunae, microinfarcts and small vessel disease in the general absence of a high burden of neurofibrillary pathology (i.e. Braak staging $<$ IV) ${ }^{26}$ or obvious other primary neurodegenerative disease, and consistent with the clinical diagnostic criteria proposed by others. ${ }^{30-33}$ An individual's condition was designated as mixed when there was pathological evidence of cerebrovascular disease with Alzheimer's-disease-type pathology, Lewy bodies or tauopathy (frontotemporal lobar degeneration or progressive supranuclear palsy). These individuals with mixed cerebrovascular disease and Alzheimer's disease had a Braak stage of V or VI and moderate to severe CERAD scores.

\section{Statistical analysis}

Univariate analysis was used to examine correlations between putative predictors and severity of depressive symptoms (rated using the GDS) at 1 year, 4 years and 8 years. These time intervals were chosen in order to ascertain whether associations between predictors and depressive symptoms changed over time, using data-sets from early, intermediate and long-term follow-up periods. Year 8 was selected as years 9 and 10 contained insufficient data for these analyses. As depressive symptoms are on an ordinal scale, we used Spearman's rank order correlation coefficient. The presence of dementia at the respective follow-up period was used as an additional independent variable.

For multivariate analyses we used multiple linear regression to examine which predictors were independently associated with severity of depressive symptomatology at each follow-up period (years 1, 4 and 8). Age and gender were entered into all models. Other putative predictors that were significant in univariate analyses at first follow-up were entered stepwise into three multivariate models ( $P=0.05$ to enter, $P=0.1$ to remove). Neuropathological findings were compared between those participants who had at least one GDS rating $\geqslant 5$ and those whose scores were always $<5$.

\section{Results}

\section{Participants}

Figure 1 shows the numbers of participants screened and recruited, and assessments of mood each year, together with deaths and withdrawals. The 355 participants recruited to the main study had a mean age of 80 years (s.d. $=4.1$ ) and $52 \%$ were male. Hospital notes revealed that 19 (5.4\%) patients had sustained a total anterior circulation stroke, $145(41 \%)$ a partial anterior circulation stroke, $116(33 \%)$ a lacunar stroke, 49 (14\%) a posterior circulation stroke and $26(7 \%)$ an unclassifiable stroke. A summary of baseline characteristics of the participants is shown in Table 1.

\section{Prevalence and incidence of depression}

Both baseline prevalence and incidence of depression varied according to method of assessment (Table 2), with the self-rated GDS giving the highest rates of depressive symptoms $(31.7 \%$ baseline prevalence), the observer-rated Cornell scale giving an intermediate rate $(9.7 \%)$ and the DSM criteria for major depression giving the lowest (1.2\%). Figure 2 shows at each year of follow-up the proportion with either major depression according to DSM-IV diagnosis or depressive symptoms above cut-offs for, GDS score $\geqslant 5$ and a Cornell score $\geqslant 10$, with the 95\% confidence intervals for the estimated percentages.

\section{Table 1 Baseline characteristics of post-stroke survivors}

\begin{tabular}{lc} 
Characteristic & Participants \\
Pre-stroke factors & \\
Age, years: mean (s.d.) & $80(4.10)$ \\
Male, $n$ (\%) & $184(51.8)$ \\
Antidepressant treatment at time of stroke, $n$ (\%) & $35(9.9)$ \\
Previous stroke, $n$ (\%) & $104(29.3)$ \\
Ischaemic heart disease, $n$ (\%) & $113(31.8)$ \\
Hypertension, $n$ (\%) & $193(54.4)$ \\
Atrial fibrillation, $n$ (\%) & $55(15.5)$ \\
Diabetes mellitus, $n$ (\%) & $29(8.2)$ \\
Hypercholesterolaemia, $n$ (\%) & $45(12.7)$ \\
Peripheral vascular disease, $n$ (\%) & $25(7.0)$ \\
Ever smoked, $n$ (\%) & $207(58.3)$ \\
Number of cardiovascular risk factors, median (IQR) & $2(1-3)$ \\
APOE $\epsilon 4$ allele (one copy), $n$ (\%) & $64 / 273(23.4)$ \\
\hline Oxford Community Stroke project stroke classification, $n$ (\%) \\
Lacunar stroke & $116(32.7)$ \\
Partial anterior circulation stroke & $145(40.8)$ \\
Total anterior circulation stroke & $19(5.4)$ \\
Posterior circulation stroke & $49(13.8)$ \\
Other & $26(7.3)$ \\
\hline Stroke outcome factors at 3 months & \\
CAMCOG total score, mean (s.d.) & $85.1(8.96)$ \\
Total CAMCOG score < 80 (CIND), $n$ (\%) & $90(25.4)$ \\
CAMCOG total memory score $\leqslant 17$ (MCI), $n$ (\%) & $50(14.1)$ \\
CAMCOG total executive function score $\leqslant 13, n$ (\%) & $168(47.3)$ \\
Geriatric Depression Score, median (IQR) & $3(1-5)$ \\
Bristol activities of daily living score & $4(1-8)$ \\
& \\
IQR, interquartile range; APOE, apolipoprotein E; CAMcoG, Cambridge Cognitive \\
Examination; CIND, cognitive impairment no dementia; MCl, mild cognitive impairment. \\
\end{tabular}




\begin{tabular}{|c|c|c|c|c|c|c|}
\hline & \multicolumn{2}{|c|}{ Major depression by DSM-IV criteria } & \multicolumn{2}{|c|}{ Geriatric Depression Scale $\geqslant 5$} & \multicolumn{2}{|c|}{ Cornell scale $\geqslant 10$} \\
\hline & $n / N$ & $\%(95 \% \mathrm{Cl})$ & $n / N$ & $\%(95 \% \mathrm{Cl})$ & $n / N$ & $\%(95 \% \mathrm{Cl})$ \\
\hline Baseline & $4 / 342$ & $1.2(0.03-2.31)$ & $110 / 347$ & $31.7(26.8-36.6)$ & $34 / 350$ & $9.7(6.61-12.8)$ \\
\hline Year 1 & $1 / 270$ & $0.4(0-1.09)$ & $79 / 262$ & $30.2(24.6-35.7)$ & $17 / 276$ & $6.2(3.32-9.00)$ \\
\hline Year 2 & $1 / 226$ & $0.4(0-1.31)$ & $60 / 227$ & $26.4(20.7-32.2)$ & $11 / 236$ & $4.7(1.97-7.35)$ \\
\hline Year 3 & 9/183 & $4.9(1.78-8.05)$ & $58 / 176$ & $33.0(26.0-39.9)$ & $16 / 185$ & $8.6(4.60-12.7)$ \\
\hline Year 4 & $4 / 149$ & $2.7(0.09-5.28)$ & $51 / 143$ & $35.7(27.8-43.5)$ & $12 / 147$ & $8.2(3.74-12.6)$ \\
\hline Year 5 & $1 / 119$ & $0.8(0-2.48)$ & $47 / 118$ & $39.8(31.0-48.7)$ & $16 / 129$ & $12.4(6.71-18.1)$ \\
\hline Year 6 & $14 / 105$ & $13.3(6.83-19.8)$ & $43 / 100$ & $43.0(33.3-52.7)$ & $16 / 109$ & $14.7(8.04-21.3)$ \\
\hline Year 7 & $7 / 73$ & $9.6(2.83-16.3)$ & $30 / 74$ & $40.5(29.4-51.7)$ & $16 / 75$ & $21.3(12.1-30.6)$ \\
\hline Year 8 & $8 / 42$ & $19.0(7.17-30.9)$ & $19 / 48$ & $39.6(25.8-53.4)$ & 9/38 & $23.7(10.2-37.2)$ \\
\hline Year 9 & $4 / 33$ & $12.1(0.99-23.3)$ & $14 / 32$ & $45.5(26.6-60.9)$ & $2 / 8$ & $25.0(5.01-55.0)$ \\
\hline Year 10 & $3 / 15$ & $20.0(0-40.2)$ & $9 / 16$ & $56.3(31.9-80.6)$ & - & - \\
\hline
\end{tabular}

The total duration of follow-up to establish depression using DSM criteria was 1245 person years (mean 3.64 years). During this time 52 episodes of major depression according to DSM-IV criteria were detected, giving an incidence of 4.18 per 100 person years. The total duration of follow-up to establish depressive symptoms using the GDS scale was 1110 person years (mean 3.20 years). During this time 410 episodes of depressive symptoms $\geqslant 5$ were detected, giving an incidence of 36.9 per 100 person years. The total duration of follow-up to establish observer-rated depression using the Cornell scale was 1203 person years (mean 3.44 years). During this time 71 episodes according to the Cornell scale $\geqslant 10$ were detected, giving an incidence of 5.90 per 100 person years.

\section{Univariate predictors of depression}

Significant univariate predictors of depressive symptoms are shown in Table 3. At first follow-up (15 months post-stroke) significant predictors included baseline GDS score (at 3 months post-stroke), lower total CAMCOG score, previous stroke, the presence of dementia at first follow-up, Bristol activities of daily living score, the presence of cognitive impairment no dementia (CIND) and number of cardiovascular risk factors. At fourth follow-up (4 years 3 months after stroke) significant predictors included baseline GDS score, lower total CAMCOG score, Bristol activities of daily living score and the presence of CIND. At eighth follow-up (8 years 3 months after stroke) only the baseline GDS score was a significant predictor of depressive symptoms.

\section{Multivariate predictors of depression}

Table 4 shows the model summaries. Baseline GDS quartile was retained as a predictor in all three models. At year 1, number of cardiovascular risk factors was also retained. At year 4, Bristol activities of daily living score and previous stroke were retained as predictors. No additional predictors were retained at year 8 , but numbers in the model were smaller $(n=32)$.

\section{Neuropathological findings}

The relative burdens of vascular and neurodegenerative disease were as expected in this group of older stroke survivors (Table 5). A large number of smaller vascular lesions distributed in the cortical and subcortical grey and white matter was observed. There was no clear evidence that the number or distribution of the infarctions were different in those with at least one depressive episode. We found only a few cases with a high burden of neurodegenerative pathology diagnostic for Alzheimer's disease (a)

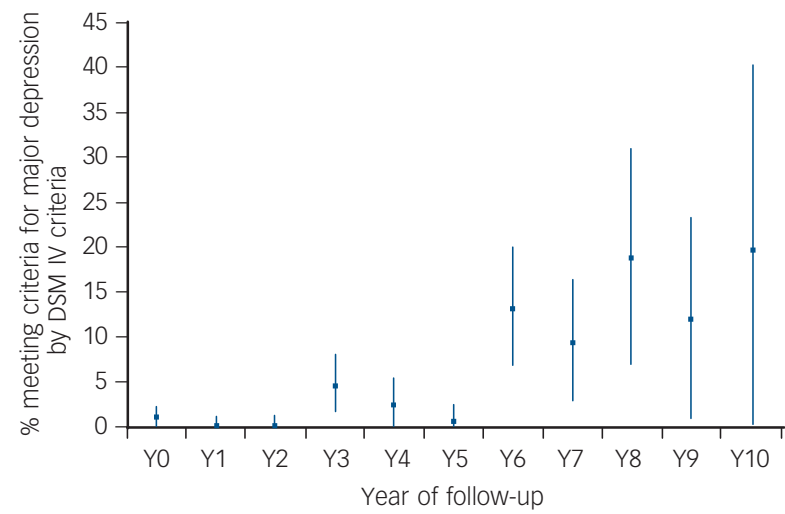

(b)

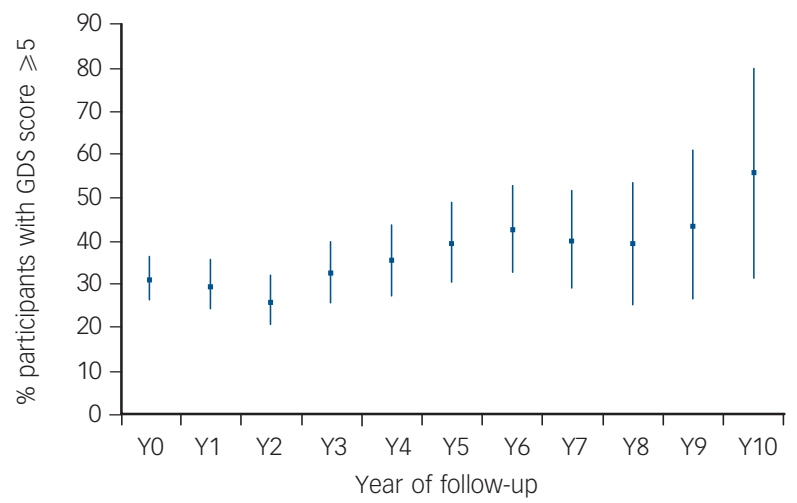

(c)

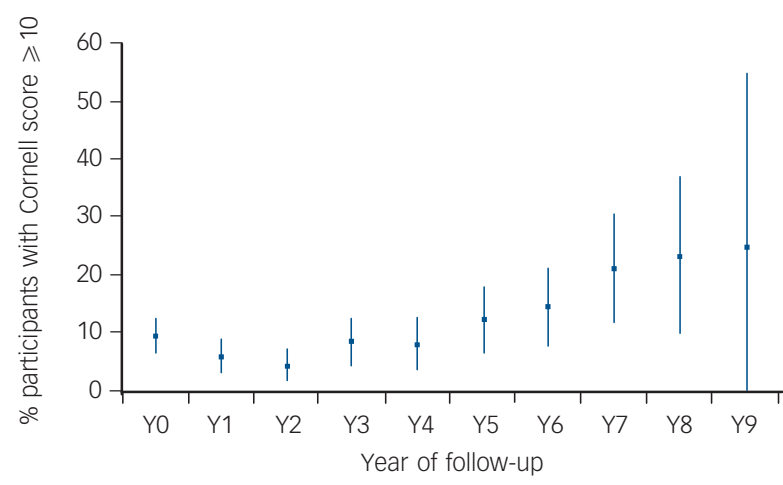

Fig. 2 Proportion of participants (a) meeting criteria for major depression by DSM-IV criteria, (b) Geriatric Depression Scale (GDS) $\geqslant 5$ and (c) Cornell score $\geqslant 10$, with error bars to show $95 \%$ confidence intervals for each proportion.

Y, year. 
Table 3 Univariate predictors of depression rated using the Geriatric Depression Scale at 1, 4 and 8 years ${ }^{a}$

\begin{tabular}{|c|c|c|c|c|c|c|}
\hline & \multicolumn{2}{|c|}{ Year 1} & \multicolumn{2}{|c|}{ Year 4} & \multicolumn{2}{|c|}{ Year 8} \\
\hline & $r$ & $P$ & $r$ & $P$ & $r$ & $P$ \\
\hline \multicolumn{7}{|l|}{ Pre-stroke factors } \\
\hline Age & -0.012 & 0.847 & 0.013 & 0.873 & 0.115 & 0.532 \\
\hline Male gender & -0.109 & 0.078 & -0.264 & 0.001 & -0.053 & 0.772 \\
\hline Antidepressant treatment at time of stroke & 0.088 & 0.163 & 0.127 & 0.135 & -0.103 & 0.576 \\
\hline Previous stroke & 0.250 & $<0.001$ & 0.110 & 0.195 & -0.167 & 0.360 \\
\hline Ischaemic heart disease & 0.007 & 0.910 & 0.093 & 0.276 & 0.193 & 0.289 \\
\hline Hypertension & 0.094 & 0.133 & 0.224 & 0.008 & 0.082 & 0.656 \\
\hline Atrial fibrillation & 0.034 & 0.586 & -0.008 & 0.923 & -0.174 & 0.342 \\
\hline Diabetes mellitus & 0.043 & 0.499 & 0.098 & 0.250 & -0.103 & 0.576 \\
\hline Hypercholesterolaemia & 0.050 & 0.429 & 0.015 & 0.860 & -0.237 & 0.191 \\
\hline Peripheral vascular disease & 0.013 & 0.833 & 0.024 & 0.776 & -0.103 & 0.576 \\
\hline Ever smoked & 0.028 & 0.666 & 0.003 & 0.972 & 0.192 & 0.294 \\
\hline Number of cardiovascular risk factors & 0.169 & 0.007 & 0.175 & 0.040 & -0.027 & 0.884 \\
\hline APOE $\epsilon 4$ allele (one copy) & 0.052 & 0.434 & 0.095 & 0.282 & -0.122 & 0.505 \\
\hline \multicolumn{7}{|l|}{ Stroke outcome factors at 3 months } \\
\hline CAMCOG total score & -0.268 & $<0.001$ & -0.260 & 0.002 & 0.308 & 0.087 \\
\hline Total CAMCOG score < 80 (CIND) & 0.178 & 0.004 & 0.243 & 0.003 & 0.301 & 0.095 \\
\hline CAMCOG total memory score $\leqslant 17(\mathrm{MCl})$ & 0.107 & 0.083 & 0.182 & 0.029 & 0.027 & 0.882 \\
\hline CAMCOG total executive function score $\leqslant 13$ & 0.105 & 0.090 & 0.190 & 0.023 & 0.293 & 0.103 \\
\hline Geriatric Depression Score: median & 0.529 & $<0.001$ & 0.474 & $<0.001$ & 0.647 & $<0.001$ \\
\hline Bristol Activities of daily living score & 0.229 & $<0.001$ & 0.244 & 0.003 & 0.266 & 0.141 \\
\hline \multicolumn{7}{|l|}{ Follow-up factor } \\
\hline Dementia at time of this assessment & 0.244 & $<0.001$ & 0.181 & 0.031 & 0.211 & 0.688 \\
\hline
\end{tabular}

or other neurodegenerative disorder. Neither Braak staging nor neuritic amyloid load was different in individuals who had experienced at least one depressive episode compared with those who had not (Table 5).

\section{Discussion}

\section{Main findings and findings from other studies}

The longer-term psychiatric sequelae of stroke have received little attention, and even for depression, which is well recognised to be common and important post-stroke. In this paper we have described the prevalence and incidence of major depression and depressive symptomatology, and identified predictors of depressive symptoms during up to 10 years of follow-up in a hospital-based cohort of survivors of first or recurrent stroke, using self-rated and observer-rated instruments as well as the researcher-rated DSM-IV criteria. We found that depression prevalence remains high and possibly increases up to 10 years. In those patients without dementia at baseline assessment
3 months post-stroke, the highest prevalence (31.7\%) and incidence (36.9 per 100 person years) of depressive symptoms was found using a self-rated scale (the GDS). These estimates are in accordance with the pooled estimates of post-stroke depression reported by Hackett et al, who found a prevalence of $34 \%(95 \%$ CI $20-39)$ at $1-6$ months post-stroke and 34\% (95\% CI 29-36) during studies following patients for more than 6 months. ${ }^{2}$ Our study is unique in the duration of its follow-up, and confirms that the frequency of depressive symptoms following a stroke remains very high even up to 10 years post-stroke. When rated by GDS, the frequency appeared to increase only slightly over time, but observer and researcher ratings did show increasing frequencies over time. As in previous studies the frequency of depression was much lower when established using DSM-IV criteria for major depression rather than the GDS, but did appear to rise over time, ranging from 0.4 to $4.9 \%$ in the first 5 years of the study to 9.6 to $20 \%$ in the latter 5 years. This is consistent with results from the OCSP, which found a frequency of depression by DSM-III criteria to be $5 \%$ in the first 12 months, rising to $18 \%$ in years 3-5. The OCSP also found much higher frequencies of

\begin{tabular}{|c|c|c|c|}
\hline & Year 1 & Year 4 & Year 8 \\
\hline \multicolumn{4}{|l|}{ Model summary } \\
\hline$n$ & 249 & 138 & 32 \\
\hline $\mathrm{R}^{2}$ & 0.305 & 0.319 & 0.416 \\
\hline Adjusted $\mathrm{R}^{2}$ & 0.293 & 0.293 & 0.354 \\
\hline Significant $F$ change & 0.001 & 0.043 & 0.000 \\
\hline \multicolumn{4}{|l|}{ Coefficients, $B(95 \% \mathrm{Cl}$ for $B)$} \\
\hline Age & $-0.011(-0.042$ to 0.020$)$ & $-0.020(-0.069$ to 0.029$)$ & $0.052(-0.061$ to 0.166$)$ \\
\hline Gender & $-0.097(-0.353$ to 0.158$)$ & $-0.313(-0.644$ to 0.019$)$ & $0.258(-0.487$ to 1.003$)$ \\
\hline Baseline Geriatric Depression Scale quartile & 0.506 (0.399 to 0.613$)$ & $0.418(0.276$ to 0.561$)$ & $0.735(0.389$ to 1.081$)$ \\
\hline Number of cardiovascular risk factors & $0.176(0.072$ to 0.280$)$ & & \\
\hline Baseline Bristol activities of daily living score & & 0.052 (0.017 to 0.087$)$ & \\
\hline Previous stroke & & $0.210(0.006$ to 0.414$)$ & \\
\hline
\end{tabular}


Table 5 Neuropathological findings according to whether any episode of depression (Geriatric Depression Scale (GDS) $\geqslant 5$ ) was recorded during follow-up

\begin{tabular}{|c|c|c|c|}
\hline Neuropathological variable & Participants without any episodes of GDS $\geqslant 5$ & Participants with at least 1 episode of GDS $\geqslant 5$ & $P$ \\
\hline$n$ & 16 & 35 & \\
\hline Brain weight, g: mean (s.d.) & $1279(143)$ & $1243(134)$ & 0.360 \\
\hline CERAD score, median (IQR) & $1(1-2)$ & $1(0-2)$ & 0.099 \\
\hline Braak score, median (IQR) & $3(2-4)$ & $3(2-3)$ & 0.193 \\
\hline CAA severity score, ${ }^{26}$ median (IQR) & $1(0-1)$ & $1(0-1.5)$ & 0.881 \\
\hline \multicolumn{4}{|l|}{ Number of infarcts, median (IQR) } \\
\hline All areas & $5(2-6.5)$ & $3.5(2-6)$ & 0.423 \\
\hline Microscopic infarcts in all areas & $3.5(2-6)$ & $2.0(1-5)$ & 0.329 \\
\hline Cortex & $1.5(1-3.5)$ & $1(0-3)$ & 0.533 \\
\hline White matter & $0(0-1)$ & $0(0-1)$ & 0.708 \\
\hline Basal ganglia and thalamus & $1(0-3)$ & $1(0-2)$ & 0.318 \\
\hline Brainstem and cerebellum & $0(0-0.5)$ & $0(0-1)$ & 0.154 \\
\hline
\end{tabular}

depression using a self-rated scale (Beck Depression Inventory). Astrom et $a l^{34}$ found an initial decrease in prevalence of depression at 1 year, compared with 3 months, but the prevalence rose again over a follow-up period of 3 years. In keeping with our results, other studies with longer-term follow-up found similar prevalence of depression; $25.8 \%$ at 5 years, ${ }^{35} 36 \%$ at 4.9 years. ${ }^{36}$

Previous studies have not used depression scales with additional information provided by the carer, although the OCSP did include an informant interview in determining depression by DSM criteria. We found that the Cornell scale gave intermediate frequencies of depression between self-rated scales and DSM criteria. This scale is particularly useful in identifying depression in patients with cognitive impairment who may be less able to communicate their symptoms, and at most time points we were able to obtain data on depressive symptoms in more patients than if we had used the self-rated scale alone.

The most consistent predictor of depression at all time points in both univariate and multivariate analyses was the baseline GDS score and this has also been a consistent finding in previous studies. This emphasises the importance of follow-up for those with significant depressive symptoms soon after stroke. Consistent with our previous finding that depressive symptoms were associated with incident dementia, cognitive impairment was also a significant univariate predictor of depression up to 4 years post-stroke, as was incident dementia at year 1 . This may be as a result of depressive symptoms being a common feature of early dementia, but also because of shared pathology and the performance effects of depression on cognitive tasks. Verdelho et $a l^{37}$ and Appelros \& Viitanen ${ }^{38}$ have both also shown that dementia is more frequent in those with depression after a stroke. In the UK, the National Institute for Health and Care Excellence recommended that in the management of acute stroke there should be screening for depression and cognitive impairment 6 weeks post-stroke. ${ }^{39}$ Guidelines on rehabilitation after stroke have also recommended that patients are screened again at 6 months and annually thereafter, ${ }^{39}$ but it is not clear how this monitoring should be carried out. We would agree that patients with early depressive symptoms, cognitive impairment or both are monitored for incident and/or recurrent depression in the long term. Given the numbers of patients to be followed up and the long-term nature of the monitoring, it seems essential that robust methods of monitoring are developed within primary care.

Prevalent depressive symptoms appeared to be strongly associated with vascular burden in the early phase after stroke, but this effect appears to weaken over prolonged follow-up. As one might expect a dose-response effect, with an increasing dose of vascular burden over time, this is perhaps surprising. However, there are alternative explanations; we know from our previous work that those with higher baseline vascular burden may have a higher mortality suggesting that the effect may have disappeared as a result of attrition of those at highest risk. Also, we did not reassess vascular risk factor burden at each follow-up and over time there may have been convergence of risk burden in surviving participants. Finally, it is possible that the vascular hypothesis is not sufficient to account for delayed episodes of post-stroke depression, and other factors such as previous experience of depression and long-term experiences of physical disability lead to negative cognitions that perpetuate depression and outweigh the effect of vascular burden. Previous long-term studies have also consistently shown the importance of dependency in activities of daily living in predicting post-stroke depression., ${ }^{3,434}$

In keeping with these observations regarding the effect of the vascular hypothesis over time, we were not able to show an increased burden of infarction or vascular disease at post-mortem in those participants who experienced at least one episode of depression. This may be because of the distance of post-mortem examinations from many of the depressive episodes.

\section{Limitations}

We deem there are a few limitations to our study. We did not have complete data on whether participants had depression prior to the stroke. However, we did include the use of antidepressants at the time of the stroke as a pre-stroke factor. As individual episodes of depression post-stroke were not followed clinically, it is possible that some episodes may have lasted more than the 12 months between follow-up assessments and will have been counted as individual episodes, thus overestimating the incidence. However, this is counterbalanced by the possibility that some episodes of depression will have emerged and resolved between annual assessments.

As a result of the availability of resources our determination of depression using DSM-IV criteria was carried out by research nurses and psychology assistants who may not have been as experienced in detecting a depressive episode as a medically qualified clinician, and we suspect that our prevalence and incidence figures may therefore be underestimated with DSM criteria. As a result of the low-event rate there were insufficient events to enable us to examine predictors of DSM-IV major depression. This is unfortunate as we have not therefore been able to address the important issue of whether clinically determined major depression has the same relationship with these predictors. 
If, as is usually assumed, major depression is the more severe subgroup, we would expect the predictors would not have been significantly different from those predicting self-rated depressive symptoms. If, however, major depression represents a different type of depression from GDS-rated depressive symptoms, then it may have different predictors.

Our cohort consists of patients who were aged at least 75 at enrolment and so the significant predictors we found may not be generalisable to a younger population. In particular, the psychosocial factors leading to depression would differ in a younger cohort, where factors such as loss of working-life roles might contribute to the onset of depression. ${ }^{3}$

\section{Implications}

In conclusion, this study has shown that the prevalence and incidence of depression and depressive symptoms in older stroke survivors are high. Significant predictors of depressive symptoms include depression at 3 months, cognitive impairment, impaired activities of daily living and in the early period, vascular risk factor burden. Post-mortem examination did not indicate an increased vascular burden in those with at least one episode of significant depressive symptoms. We recommend that long-term monitoring of mood is undertaken in those at high risk of depression, and although control of risk factor burden may be important, particularly for the maintenance of cognition, long-term psychosocial and functional support are likely to be key components of the management of these patients.

\footnotetext{
Louise M. Allan, PhD, MRCP, Elise N. Rowan, PhD, Alan J. Thomas, PhD,
FRCPsych, Tuomo M. Polvikoski, MD, John T. O'Brien, DM, Raj N. Kalaria, PhD, FRCPsych, Institute for Ageing and Health, Newcastle University, Newcastle upon Tyne, UK

Correspondence: Raj N. Kalaria, Institute for Ageing and Health, Newcastle University Campus for Ageing and Vitality, Newcastle upon Tyne NE4 5PL, UK. Email: r.n.kalaria@ncl.ac.uk

First received 27 Feb 2013, final revision 20 Jun 2013, accepted 5 Sep 2013
}

\section{Funding}

Our work is supported by grants from the UK Medical Research Council (MRC, G0500247), Newcastle Centre for Brain Ageing and Vitality (Biotechnology and Biological Sciences Research Council, Engineering and Physical Sciences Research Council, Economic and Research Council, Engineering and Physical Sciences Research Council, Economic and
Social Research Council and MRC, Lifelong Health and Wellbeing) and Alzheimer's Research UK (ARUK). Tissue for this study was collected by the Newcastle Brain Tissue Resource, which is funded in part by a grant from the UK MRC (G0400074), by the Newcastle NIHR Biomedical Research Centre in Ageing and Age Related Diseases award to the Newcastle upon Tyne Hospitals NHS Foundation Trust, and by a grant from the Alzheimer's Society and ARUK as part of the Brains for Dementia Research Project.

\section{Acknowledgements}

We are very grateful to the patients, families and clinical house staff for their cooperation in the investigation of this study. We thank Michelle Widdrington, Carein Todd, Jean Scott Deborah Lett and Anne Nicholson for assistance in managing and screening the cohort. We are indebted to Professors Clive Ballard and Rose Anne Kenny for guiding this longitudinal study in its early phases

\section{References}

1 Ayerbe L, Ayis S, Wolfe CDA, Rudd AG. Natural history, predictors and outcomes of depression after stroke: systematic review and meta-analysis. Br J Psychiatry 2013; 202: 14-21.

2 Hackett ML, Yapa C, Parag V, Anderson CS. Frequency of depression after stroke: a systematic review of observational studies. Stroke 2005; 36: 1330-40.

3 Ayerbe L, Ayis S, Rudd AG, Heuschmann PU, Wolfe CD. Natural history, predictors, and associations of depression 5 years after stroke: the South London Stroke Register. Stroke 2011; 42: 1907-11.
4 Sharpe M, Hawton K, Seagroatt V, Bamford J, House A, Molyneux A, et al. Depressive disorders in long-term survivors of stroke. Associations with demographic and social factors, functional status, and brain lesion volume. Br J Psychiatry 1994; 164: 380-6.

5 Hackett ML, Anderson CS. Predictors of depression after stroke: a systematic review of observational studies. Stroke 2005; 36: 2296-301.

6 Hackett ML, Anderson CS. Frequency, management, and predictors of abnormal mood after stroke: the Auckland Regional Community Stroke (ARCOS) study, 2002 to 2003. Stroke 2006; 37: 2123-8.

7 Valkanova V, Ebmeier KP. Vascular risk factors and depression in later life: a systematic review and meta-analysis. Biol Psychiatry 2013; 73: 406-13.

8 Allan LM, Rowan EN, Firbank MJ, Thomas AJ, Parry SW, Polvikoski TM, et al. Long term incidence of dementia, predictors of mortality and pathological diagnosis in older stroke survivors. Brain 2012; 134: 3716-27.

9 Desmond DW, Moroney JT, Paik MC, Sano M, Mohr JP, Aboumatar S, et al. Frequency and clinical determinants of dementia after ischemic stroke. Neurology 2000; 54: 1124-31.

10 Folstein MF, Folstein SE, McHugh PR. Mini-mental state. A practical method for grading the cognitive state of patients for the clinician. J Psychiatr Res 1975; 12: 189-98.

11 World Health Organization. Cerebrovascular Disorders: A Clinical and Research Classification. WHO, 1978.

12 American Psychiatric Association. Diagnostic and Statistical Manual of Mental Disorders (3rd edn, revised) (DSM-III-R). APA, 1987.

13 Ballard C, Rowan E, Stephens S, Kalaria R, Kenny RA. Prospective follow-up study between 3 and 15 months after stroke: improvements and decline in cognitive function among dementia-free stroke survivors $>75$ years of age. Stroke 2003; 34: 2440-4.

14 Ballard C, Stephens S, Kenny R, Kalaria R, Tovee M, O'Brien J. Profile of neuropsychological deficits in older stroke survivors without dementia. Dement Geriatr Cogn Disord 2003; 16: 52-6.

15 Allan LM, Ballard CG, Rowan EN, Kenny RA. Incidence and prediction of falls in dementia: a prospective study in older people. PLOS One 2009; 4: e5521.

16 Ballard CG, Morris CM, Rao H, O'Brien JT, Barber R, Stephens S, et al. APOE epsilon4 and cognitive decline in older stroke patients with early cognitive impairment. Neurology 2004; 63: 1399-402.

17 Bamford J, Sandercock P, Dennis M, Burn J, Warlow C. Classification and natural history of clinically identifiable subtypes of cerebral infarction. Lancet 1991; 337: 1521-6.

18 Bucks RS, Ashworth DL, Wilcock GK, Siegfried K. Assessment of activities of daily living in dementia: development of the Bristol Activities of Daily Living Scale. Age Ageing 1996; 25: 113-20.

19 Roth M, Tym E, Mountjoy CQ, Huppert FA, Hendrie H, Verma S, et al. CAMDEX. A standardised instrument for the diagnosis of mental disorder in the elderly with special reference to the early detection of dementia. Br J Psychiatry 1986; 149: 698-709.

20 Petersen RC, Smith GE, Waring SC, Ivnik RJ, Kokmen E, Tangelos EG. Aging, memory, and mild cognitive impairment. Int Psychogeriatr 1997; 9 (suppl 1): 65-9.

21 Almeida OP, Almeida SA. Short versions of the geriatric depression scale: a study of their validity for the diagnosis of a major depressive episode according to ICD-10 and DSM-IV. Int J Geriatr Psychiatry 1999; 14: 858-65.

22 American Psychiatric Association. Diagnostic and Statistical Manual of Mental Disorders (4th edn) (DSM-IV). APA, 1994.

23 Alexopoulos GS, Abrams RC, Young RC, Shamoian CA. Cornell Scale for Depression in Dementia. Biol Psychiatry 1988; 23: 271-84.

24 Teodorczuk A, Firbank MJ, Pantoni L, Poggesi A, Erkinjuntti T, Wallin A, et al. Relationship between baseline white-matter changes and development of late-life depressive symptoms: 3-year results from the LADIS study. Psychol Med 2010; 40: 603-10.

25 Teodorczuk A, O'Brien JT, Firbank MJ, Pantoni L, Poggesi A, Erkinjuntti T, et al. White matter changes and late-life depressive symptoms. Longitudinal study. Br J Psychiatry 2007; 191: 212-7.

26 Kalaria RN, Kenny RA, Ballard CG, Perry R, Ince P, Polvikoski T. Towards defining the neuropathological substrates of vascular dementia. J Neurol Sci 2004; 226: 75-80.

27 Ihara M, Polvikoski TM, Hall R, Slade JY, Perry RH, Oakley AE, et al. Quantification of myelin loss in frontal lobe white matter in vascular dementia, Alzheimer's disease, and dementia with Lewy bodies. Acta Neuropathol 2010; 119: 579-89.

28 Mirra SS, Heyman A, McKeel D, Sumi SM, Crain BJ, Brownlee LM, et al. The Consortium to Establish a Registry for Alzheimer's Disease (CERAD). Part II. 
Standardization of the neuropathologic assessment of Alzheimer's disease. Neurology 1991; 41: 479-86.

29 Braak H, Alafuzoff I, Arzberger T, Kretzschmar H, Del Tredici K. Staging of Alzheimer disease-associated neurofibrillary pathology using paraffin sections and immunocytochemistry. Acta Neuropathol 2006; 112: 389-404.

30 Chui HC, Victoroff Jl, Margolin D, Jagust W, shankle R, Katzman R. Criteria for the diagnosis of ischemic vascular dementia proposed by the State of California Alzheimer's disease diagnostic and treatment centers. Neurology 1992; 42: 473-80.

31 Chui HC, Mack W, Jackson JE, Mungas D, Reed BR, Tinklenberg J, et al. Clinical criteria for the diagnosis of vascular dementia: a multicenter study of comparability and interrater reliability. Arch Neurol 2000; 57: 191-6.

32 Gold G, Bouras C, Canuto A, Bergallo MF, Herrmann FR, Hof PR, et al. Clinicopathological validation study of four sets of clinical criteria for vascular dementia. Am J Psychiatry 2002; 159: 82-7.

33 Roman GC, Tatemichi TK, Erkinjuntti T, Cummings JL, Masdeu JC, Garcia JH et al. Vascular dementia: diagnostic criteria for research studies. Report of the NINDS-AIREN International Workshop. Neurology 1993; 43: 250-60.
34 Astrom M, Adolfsson R, Asplund K. Major depression in stroke patients. A 3-year longitudinal study. Stroke 1993; 24: 976-82.

35 Chausson N, Olindo S, Cabre P, Saint-Vil M, Smadja D. Five-year outcome of a stroke cohort in Martinique, French West Indies: Etude Realisee en Martinique et Centree sur I'Incidence des Accidents vasculaires cerebraux Part 2. Stroke 2010; 41: 594-9.

36 Wilkinson PR, Wolfe CD, Warburton FG, Rudd AG, Howard RS, Ross-Russell RW, et al. A long-term follow-up of stroke patients. Stroke 1997; 28: 507-12.

37 Verdelho A, Henon H, Lebert F, Pasquier F, Leys D. Depressive symptoms after stroke and relationship with dementia: a three-year follow-up study. Neurology 2004; 62: 905-11.

38 Appelros $\mathrm{P}$, Viitanen $\mathrm{M}$. Prevalence and predictors of depression at one year in a Swedish population-based cohort with first-ever stroke. J Stroke Cerebrovasc Dis 2004; 13: 52-7.

39 National Institute for Health and Care Excellence. Stroke Rehabilitation: Long-Term Rehabilitation after Stroke. NICE, 2013.

\section{poems \\ by doctors}

\section{Black Dog}

\section{Peter Wells}

Someone's switched off the sunlight;

my river's run dry.

Amber streaks the velvet darkness;

there's no echo to my call.

A loved one craves affection;

there's no reaching her'

I've forgotten the language:

mute messenger of love!

Peter Wells is a psychiatrist who worked with disturbed young people for over 25 years. Two of his poems have been published as 'Extras', Strategy (November 2010) and Scored (July 2011). (c) 2012 Peter Wells. 